\title{
Regulation of Cdh1-APC Function in Axon Growth by Cdh1 Phosphorylation
}

\author{
Mai Anh Huynh, ${ }^{1,2,3}$ Judith Stegmüller, ${ }^{1}$ Nadia Litterman, ${ }^{1,4}$ and Azad Bonni ${ }^{1,2,4}$ \\ ${ }^{1}$ Department of Pathology, ${ }^{2}$ Program in Biology and Biomedical Sciences, ${ }^{3}$ Harvard University-Massachusetts Institute of Technology Division of Health, \\ Sciences, and Technology, and 4 Program in Neuroscience, Harvard Medical School, Boston, Massachusetts 02115
}

The ubiquitin ligase Cdh1-anaphase promoting complex (Cdh1-APC) plays a key role in the control of axonal morphogenesis in the mammalian brain, but the mechanisms that regulate neuronal Cdh1-APC function remain incompletely understood. Here, we have characterized the effect of phosphorylation of Cdh1 at cyclin-dependent kinase (Cdk) sites on Cdh1-APC function in neurons. We replaced nine conserved sites of Cdk-induced Cdh1 phosphorylation with alanine (9A) or aspartate (9D) to mimic hypo- or hyperphosphorylation, respectively. We found that the $9 \mathrm{~A}$ mutation triggered the proteasome-dependent degradation of Cdh1, and conversely the 9D mutation stabilized Cdh1 in neuronal cells. However, the phosphomimic 9D Cdh1 protein failed to associate with the APC core protein Cdc27. In addition, whereas wild-type and 9A Cdh1 predominantly localized to the nucleus, the 9D Cdh1 protein accumulated in the cytoplasm in neurons. Importantly, in contrast to wild-type and 9A Cdh1, the 9D Cdh1 mutant failed to inhibit axon growth in primary cerebellar granule neurons. Collectively, our results suggest that phosphorylation of neuronal $\mathrm{Cdh} 1$ at $\mathrm{Cdk}$ sites triggers the stabilization of an inactive form of Cdh1 that accumulates in the cytoplasm, leading to the inhibition of Cdh1-APC function in the control of axon growth. Thus, phosphorylation of Cdh1 may represent a critical mechanism regulating Cdh1-APC function in the nervous system.

\section{Introduction}

The E3 ubiquitin ligase activity of the anaphase-promoting complex (APC) is critical for coordinating cell cycle transitions (Peters, 2006; Sullivan and Morgan, 2007). The association of the APC with one of two activating subunits, Cdh1 or Cdc20, enables this highly conserved, multi-subunit complex to target specific substrates for proteasomal degradation during distinct phases of the cell cycle (Peters, 2006; Sullivan and Morgan, 2007). Recent studies have defined new functions for the APC in postmitotic neurons (Stegmüller and Bonni, 2005; Kim and Bonni, 2007; Kim et al., 2009). In particular, Cdh1-APC controls axon growth and patterning in the mammalian brain (Konishi et al., 2004). Neuronal Cdh1-APC may also regulate synapse development and plasticity (Juo and Kaplan, 2004; van Roessel et al., 2004; Li et al., 2008) and neuronal survival (Almeida et al., 2005). Thus, Cdh1APC appears to orchestrate diverse aspects of neuronal development and function.

The mechanisms by which Cdh1-APC controls axonal morphogenesis are beginning to be characterized. Structure-function analyses of Cdh1 have revealed that Cdh1-APC operates in the

\footnotetext{
Received Nov. 5, 2008; revised Feb. 9, 2009; accepted March 2, 2009.

This work was supported by National Institutes of Health (NIH) Grant NS051255 (A.B.), the Harvard Medical School-Merck-Sponsored Research Program (A.B.), a Charles A. King Trust (Bank of America, Cotrustee) Postdoctoral Fellowship (J.S.), the National Science Foundation (N.L.), and NIH Training Grant GM077226 (M.A.H.). We thank L. H. Tsai for providing p 25 and Cdk5 expression plasmids and members of the Bonni laboratory for helpful discussions.

Correspondence should be addressed Azad Bonni, Harvard Medical School, New Research Building, Bonni Laboratory, Room 858, 77 Avenue Louis Pasteur, Boston, MA 02115. E-mail: azad_bonni@hms.harvard.edu.

J. Stegmüller's present address: Max Planck Institute of Experimental Medicine, Hermann-Rein-Strasse 3, 37075 Göttingen, Germany.

DOI:10.1523/JNEUROSC1.5329-08.2009

Copyright $\odot 2009$ Society for Neuroscience $\quad$ 0270-6474/09/294322-06\$15.00/0
}

nucleus in neurons to inhibit axon growth (Stegmüller et al., 2006). The identification of the transcriptional regulators SnoN and Id 2 as physiologically relevant substrates of neuronal Cdh1APC suggests that this ubiquitin ligase controls programs of gene expression that are dedicated to the growth of axons (Lasorella et al., 2006; Stegmüller et al., 2006; Ikeuchi et al., 2009).

How Cdh1-APC function is controlled in neurons remains to be elucidated. The TGF $\beta-\operatorname{Smad} 2$ signaling pathway stimulates the ability of neuronal Cdh1-APC to trigger the degradation of SnoN and thereby inhibit axon growth (Stegmüller et al., 2008). Activated Smad2 may facilitate the assembly of a tripartite complex composed of Smad2, Cdh1, and SnoN, potentially promoting the interaction of Cdh1-APC with the substrate SnoN (Pot and Bonni, 2008). In addition to mechanisms of protein-protein interaction, neuronal Cdh1-APC function might also be regulated by phosphorylation of Cdh1 (Maestre et al., 2008). However, whether Cdh1 phosphorylation plays a role in Cdh1-APC regulation of axon growth remained to be addressed.

In mitotic cells, Cdh1-APC activity is tightly regulated by cyclin-dependent kinase (Cdk)-induced phosphorylation of Cdh1. The mitotic Cdk-induced phosphorylation of Cdh1 dissociates Cdh1 from the APC (Peters, 2006). Phosphorylation of Cdh 1 may occur on as many as 11 sites in budding yeast and nine conserved residues in proliferating mammalian cells (Zachariae et al., 1998; Jaspersen et al., 1999; Kramer et al., 2000). Phosphorylation at four of these sites (serines 40,151, and 163 and threonine 121) reportedly promotes the nuclear export of mammalian Cdh1 (Zhou et al., 2003). These observations led us to question the consequence of Cdh1 phosphorylation at Cdk sites on Cdh1APC regulation of axon growth in mammalian neurons.

Here, we find that Cdh1 phosphorylation may represent a key 
A

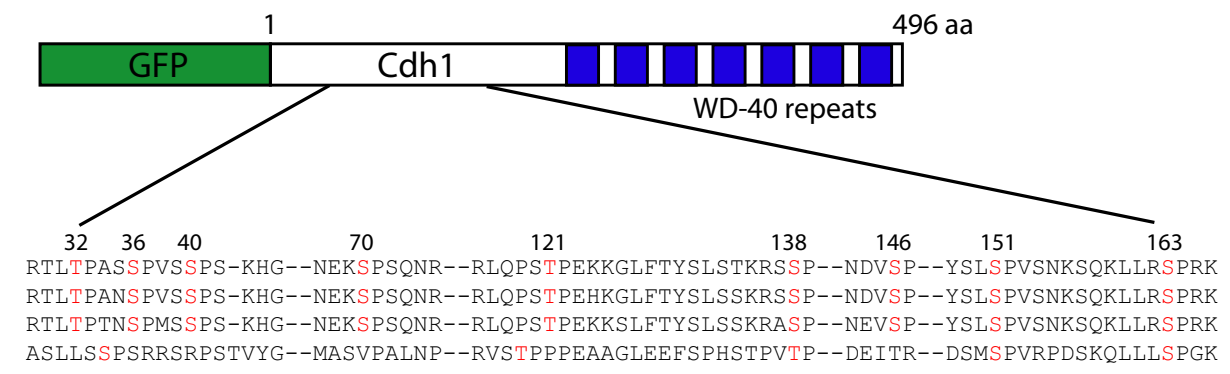

B

$$
\begin{aligned}
& \text { cdh1_human } \\
& \text { cdh1_mouse } \\
& \text { cdh1_xenopus } \\
& \text { cdh1_yeast }
\end{aligned}
$$

$\mathrm{E}$

C

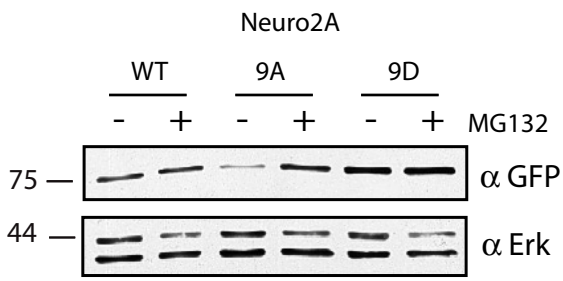

Neurons

D

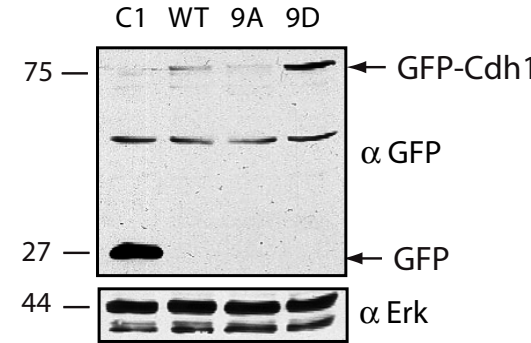

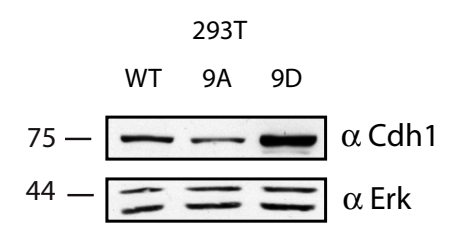

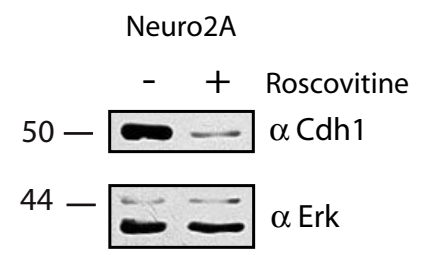

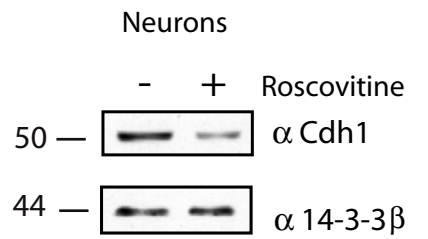

$\mathrm{F}$

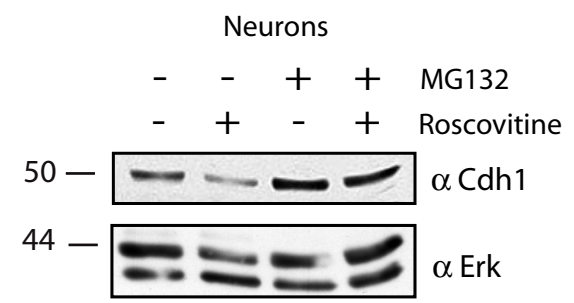

Figure 1. Cdh1 phosphorylation at Cdk sites promotes (dh1 stability. A, Schematic of nine conserved sites of potential Cdk phosphorylation in Cdh1 (S/T-P), numbered according to human Cdh1. $B$, Lysates of 293T cells transfected with GFP-Cdh1 WT, 9A, or 9D were immunoblotted using a polyclonal antibody to Cdh1 or Erk, the latter to serve as a loading control. Quantitation of Cdh1 levels normalized by Erk revealed that the levels of the $9 \mathrm{D}$ and $9 \mathrm{~A}$ mutant proteins were, respectively, increased by $122 \%$ and reduced by $57 \%$ relative to wild-type Cdh1 in $293 \mathrm{~T}$ cells (average of 4 experiments). C, Lysates of Neuro2A cells transfected with GFP-Cdh1 WT, 9A, or 9D and treated with MG132 (5 $\mu \mathrm{M}$ ) or the vehicle control DMSO were immunoblotted for GFP or Erk. The levels of the $9 \mathrm{D}$ and $9 \mathrm{~A}$ mutant proteins were, respectively, increased by $150 \%$ and reduced by $37 \%$ relative to wild-type Cdh1 in Neuro2A cells (average of 2 experiments). MG132 treatment, respectively, increased WT, 9A, and 9D levels by 80, 134, and 9\%. D, Lysates of granule neurons transfected with pEGFP-C1, GFP-Cdh1 WT, GFP-Cdh1 9A, or GFP-Cdh1 9D were immunoblotted for GFP or Erk. The $9 D$ Cdh1 mutant is expressed at higher levels than wild-type $\mathrm{Cdh} 1$, which is higher than the $9 \mathrm{~A} C \mathrm{Cdh} 1$ mutant in primary granule neurons. $E$, Lysates of Neuro2A cells or granule neurons treated with roscovitine $(20 \mu \mathrm{M})$ or DMSO were immunoblotted using a monoclonal antibody to $\mathrm{Cdh} 1$ and the Erk or $14-3-3 \beta$ antibody. Roscovitine reduces $\mathrm{Cdh} 1$ levels by $60 \%$ in both Neuro2A and neurons (average of 2 experiments each). $\boldsymbol{F}$, Lysates of granule neurons treated with MG132 $(10 \mu \mathrm{M})$, roscovitine $(20 \mu \mathrm{M})$, both MG132 and roscovitine, or DMSO were immunoblotted using the Erk antibody or the Cdh1 monoclonal antibody. Roscovitine decreased (dh1 levels by 50\%, whereas MG132 increased Cdh1 levels by almost twofold (average of 2 experiments). The combination of MG132 and roscovitine treatment restored Cdh1 levels to 1.3-fold above control Cdh1 levels.

mode of regulation of Cdh1-APC function in neurons. Our results suggest that phosphorylation of Cdh1 at nine conserved Cdk sites disrupts the association of Cdh1 with the APC, leading to the stabilization of an inactive form of Cdh1 in the cytoplasm and consequent inhibition of Cdh1-APC function in limiting the growth of axons.

\section{Materials and Methods}

Reagents. The 9A and 9D mutations in green fluorescent protein (GFP)Cdh1 (Stegmüller et al., 2006) were generated using Quik-Change sitedirected mutagenesis (Stratagene) to mutate the Cdh1 sites shown in Figure $1 A$. The GFP (Invitrogen), Erk 1/2 (Cell Signaling Technology), monoclonal Cdh1 (Genetex), polyclonal Cdh1 (Zymed), Cdc27 (Santa Cruz Biotechnology), Discosoma red (DsRed) (Clontech), and $\beta$-galactosidase ( $\beta$-gal) (Promega) antibodies were purchased. MG132
(carbobenzoxy-L-leucyl-L-leucyl-L-leucinal) (Sigma) and roscovitine (Calbiochem) were purchased.

Immunoprecipitations and immunoblotting analyses. Immunoprecipitation and immunoblotting analyses were done as described previously (Kim et al., 2009).

Glycerol density gradient. Using a protocol modified from the study of Zachariae et al. (1996), lysates of granule neurons were prepared in buffer A (1\% Triton X-100, $150 \mathrm{~mm} \mathrm{NaCl,} 1$ mm EDTA, and $100 \mathrm{~mm}$ Tris, pH 7.2), layered on $15-35 \%$ glycerol density gradients in buffer A, and centrifuged for $18 \mathrm{~h}$ at 28,000 rpm in a Beckman Coulter SW60 rotor. Fractions were analyzed by SDS-PAGE and immunoblotting for Cdc27 and Cdh1.

Primary neuron cultures and transfection. Granule neurons were prepared from postnatal day 6 Long-Evans rat pups and transfected either $8 \mathrm{~h}$ or $2 \mathrm{~d}$ in vitro after plating using a modified calcium phosphate 
method as described previously (Konishi et al., 2004) with indicated plasmids together with either DsRed or $\beta$-gal expression plasmid to visualize transfected neurons. To rule out the possibility that the effects of RNA interference (RNAi) or protein expression on axonal length were attributable to any effect of these manipulations on cell survival, the anti-apoptotic protein $\mathrm{Bcl}-\mathrm{x}_{\mathrm{L}}$ was coexpressed in the morphology experiments. The expression of $\mathrm{Bcl}-\mathrm{x}_{\mathrm{L}}$ has little or no effect on axonal length (Konishi et al., 2004). Neurons were subjected to immunocytochemistry using a GFP, DsRed, and/or $\beta$-gal antibody.

Axon growth assay and morphometry. Axon length morphometry was done as described previously (Gaudilliere et al., 2004; Konishi et al., 2004). Briefly, images of transfected DsRed-positive neurons were captured in a blinded manner using a Nikon Eclipse TE2000 epifluorescence microscope. Axon length was measured using SPOT imaging software. Cellular localization was quantified by counting the percentage of transfected $\beta$-gal-positive neurons harboring exclusively nuclear or nuclear and strong cytoplasmic GFP staining.

\section{Results}

The Cdk-induced phosphorylation of Cdh1 has been characterized in yeast and proliferating non-neural mammalian cells ( $\mathrm{Pe}$ ters, 2006; Sullivan and Morgan, 2007). Recent evidence suggests that Cdh1 is also phosphorylated in mammalian neurons (Maestre et al., 2008). However, the role of Cdh1 phosphorylation in Cdh1-APC function in axon growth remained unknown. To investigate the role of Cdh1 phosphorylation in neuronal cells, we generated plasmids encoding a GFP-Cdh1 fusion protein mutated to alanine (9A) or aspartate (9D) at nine sites of Cdk phosphorylation conserved from Xenopus to human to mimic hypoor hyper-phosphorylation, respectively (Fig. $1 A$ ).

We first expressed the wild-type (WT) and phosphorylation mutants of Cdh1 in 293T cells and mouse Neuro2A neuroblastoma cells. Remarkably, we found a clear and consistent difference in the protein levels of hypo- versus hyper-phosphorylation mutant Cdh1 in these cells (Fig. $1 B, C$ ). The phosphomimic GFP-Cdh1 9D was expressed at higher levels than GFP-Cdh1 WT, which in turn was expressed at higher levels than GFP-Cdh1 9A in both $293 \mathrm{~T}$ cells and neuronal cells (Fig. 1B,C). To determine the basis for the difference in the level of expression of wild-type and phosphorylation mutants of Cdh1 protein, we assessed the effect of treatment of cells with the proteasome inhibitor MG132 on the levels of the Cdh1 proteins. The levels of wild-type and both mutant Cdh1 proteins increased in 293T cells upon exposure to MG132, suggesting that Cdh1 levels are exquisitely regulated by proteasome-dependent degradation (data not shown). Notably, we also found that the levels of GFP-Cdh1 9A, and to a lesser extent GFP-Cdh1 WT, were elevated in Neuro2A cells upon exposure to MG132 (Fig. 1C). In contrast, the levels of GFP-Cdh1 9D, which were basally high, were not further elevated in Neuro2A cells treated with MG132 (Fig. 1C). These results suggest that the phosphorylation of Cdh1 at Cdk sites stabilizes Cdh1 protein, whereas dephosphorylation destabilizes Cdh1 in neuronal cells. A caveat that cannot be ruled out is that mutation of Cdh1 at Cdk sites might trigger conformational changes in Cdh1 protein that account for the observed changes in Cdh1 stability.

We next determined the effect of Cdh1 phosphorylation at Cdk sites on the stability of Cdh1 in primary neurons. In agreement with results in $293 \mathrm{~T}$ and Neuro2A cells, the 9D Cdh1 mutant was expressed at higher levels, whereas the 9A Cdh1 mutant was expressed at lower levels, than wild-type Cdh1 upon expression in primary rat cerebellar granule neurons (Fig. $1 D$ ). In addition, we found that MG132 treatment was sufficient to elevate endogenous Cdh1 protein levels in primary neurons (Fig. $1 \mathrm{~F}$ ). To
A
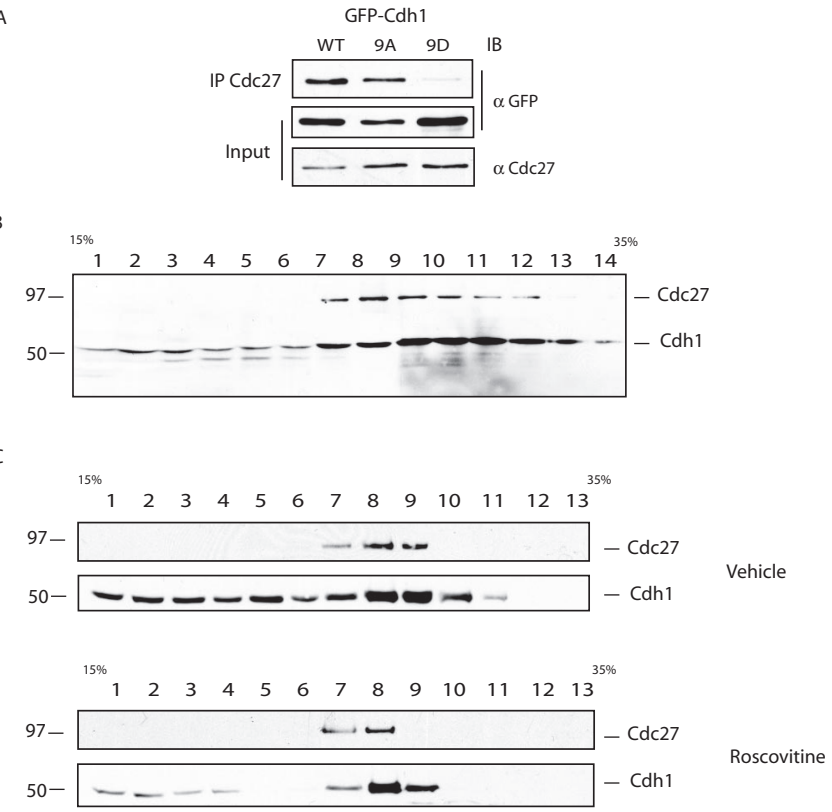

D

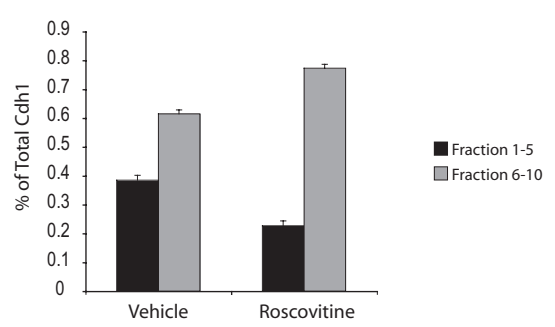

Figure 2. Cdh1 phosphorylation at Cdk sites disrupts the association of Cdh1 with the APC core protein Cdc27. A, The APC core protein (dc27 was immunoprecipitated (IP) from lysates of 293T cells expressing GFP-Cdh1 WT, 9A, or 9D followed by immunoblotting with the GFP or Cdc27 antibody. The 9D mutation, but not the 9A mutation, disrupts the ability of Cdh1 to form a complex with the APC core protein Cdc27. B, Glycerol density gradient (15-35\%) centrifugation of extracts was performed on cerebellar granule neurons, and fractions were immunoblotted using a Cdc27 antibody and a monoclonal antibody to Cdh1. Endogenous C $\mathrm{dh} 1$ is found in two pools in neurons: a pool that comigrates with $\mathrm{Cdc} 27$ and a pool that is free of $\mathrm{Cdc} 27 . \mathbf{C}, \mathbf{D}$, Glycerol density gradient (15-35\%) centrifugation of extracts was performed on cerebellar granule neurons treated for $11 \mathrm{~h}$ with MG132 (10 $\mu \mathrm{m})$ and either roscovitine (20 $\mu \mathrm{m})$ or the vehicle control DMSO. Roscovitine treatment increased the pool of Cdh1 cofractionating with Cdc27 from 62 to $77 \%$ but decreased the free pool of Cdh1 from 38 to $23 \%$ ( $n=3$; ANOVA with Fisher's PLSD post hoc test, $p<0.0001)$.

test the hypothesis that Cdk phosphorylation stabilizes Cdh1 protein, we assessed the effect of inhibiting endogenous Cdk activity on the levels of endogenous Cdh1 in neuronal cells, including primary granule neurons. Exposure of Neuro2A cells and primary granule neurons to the $\mathrm{Cdk}$ inhibitor roscovitine robustly reduced the levels of endogenous Cdh1 (Fig. 1E,F). The roscovitine-induced reduction in Cdh1 levels was reversed upon exposure of neurons to MG132, suggesting that Cdk inhibition triggers the proteasomal degradation of Cdh1 in neurons (Fig. $1 \mathrm{~F}$ ). Together, these results suggest that phosphorylation of Cdh1 at Cdk sites inhibits the degradation of Cdh1 by the ubiquitinproteasome pathway in neurons.

What is the consequence of phosphorylation-induced stabilization of Cdh1 on Cdh1-APC function? To address this question, we first determined the effect of Cdh1 phosphorylation on its ability to associate with the APC. We expressed wild-type and phosphorylation mutants of GFP-Cdh1 in cells and assessed their ability to form a physical complex with the APC core protein 
A
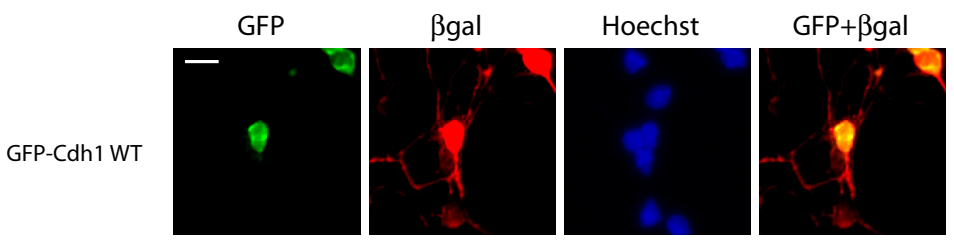

GFP-Cdh1 9D
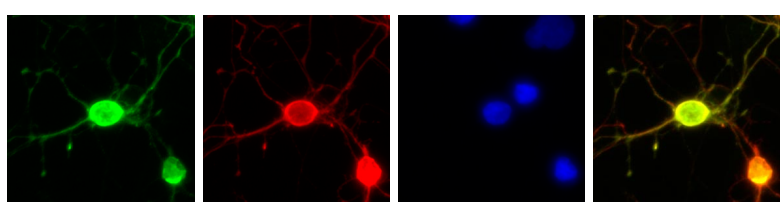

GFP-Cdh1 9A
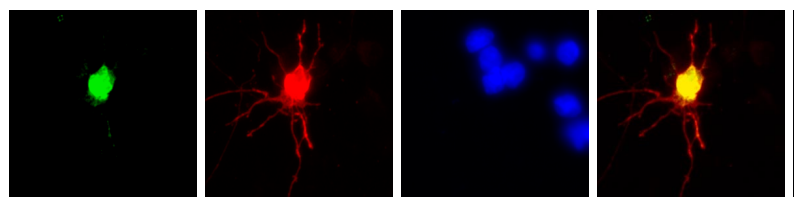

B

$$
\text { C }
$$

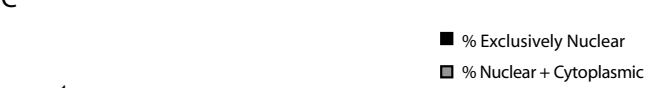

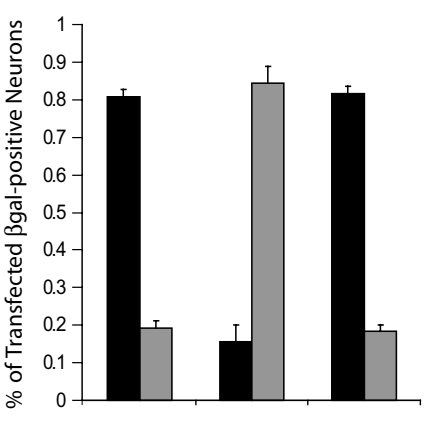

GFP-Cdh1 WT GFP-Cdh1 9D GFP-Cdh19A
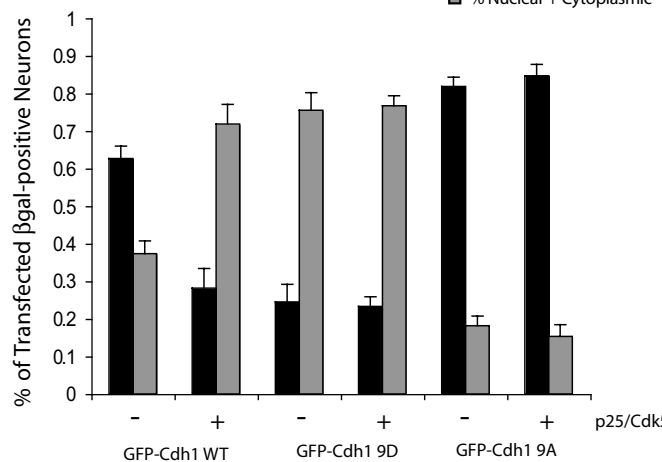

Figure 3. Cdh1 phosphorylation at C $d k$ sites promotes the cytoplasmic accumulation of $C d h 1$ in granule neurons. $A$, Granule neurons transfected with an expression plasmid encoding GFP-Cdh1 WT, 9D, or 9A, together with the $\beta$-gal expression plasmid were subjected to immunocytochemistry with an antibody to $\beta$-gal or GFP and the DNA dye Hoechst. Scale bar, $10 \mu \mathrm{m}$. $\boldsymbol{B}$, Wild-type and the $9 \mathrm{~A} C \mathrm{Cdh} 1$ mutant were exclusively nuclear in $\sim 80 \%$ of neurons. However, the $9 \mathrm{D}$ mutation significantly reduced the number of neurons displaying exclusively nuclear localization (ANOVA with Fisher's PLSD post hoc test, $p<0.0001$ ). A total of 788 neurons were analyzed. $\boldsymbol{C}$, Neurons transfected as in $\boldsymbol{A}$ together with expression plasmids encoding p25 and $\mathbf{C d k} 5$ or their vector control pCMV5 were analyzed as in $\boldsymbol{A}$ and $\boldsymbol{B}$. The percentage of neurons in which Cdh1 appeared in the cytoplasm increased from $<40$ to $>70 \%$ upon p25/Cdk5 expression (ANOVA with Fisher's PLSD post hoc test, $p<0.0001$ ). In contrast, $9 A$ Cdh1 and 9D Cdh1 remained predominantly nuclear or nuclear and cytoplasmic, respectively, upon p25/Cdk5 expression. A total of 2500 neurons were analyzed.

Cdc27 by immunoblotting Cdc27 immunoprecipitates with the GFP antibody. The 9A Cdh1 mutant associated with Cdc27 as effectively as wild-type Cdh1 (Fig. 2 A). In contrast, the phosphomimic 9D Cdh1 mutant failed to associate effectively with Cdc27 (Fig. 2A). These results suggest that phosphorylation of Cdh1 stabilizes an inactive form of Cdh 1 that is unable to complex with the APC. Consistent with these results, Cdk phosphorylation of Cdh1 in yeast and Xenopus extracts triggers the dissociation of Cdh1 from the APC (Zachariae et al., 1998; Jaspersen et al., 1999; Kramer et al., 2000). Thus, phosphorylation of Cdh1 at Cdk sites appears to represent a highly evolutionarily conserved mechanism that regulates the association of Cdh1 with the APC.

If the association of Cdh1 with the APC represents a mode of regulation in mammalian neurons, one would predict that Cdh1 is present in two pools, a pool that is bound to the APC and a pool of Cdh1 that is not in complex with the APC. To test this predic-
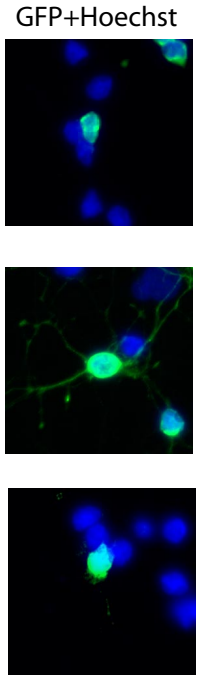

tion, we separated granule neuron lysates on a glycerol gradient and subjected the different fractions to immunoblotting with antibodies to Cdh1 and Cdc27. We found that endogenous Cdh1 appears in two peaks, one low-percentage and the other high-percentage glycerol fractions. The APC core protein Cdc27 was found in a similar peak as the high-percentage glycerol Cdh1 peak but not in the lowpercentage glycerol Cdh1 peak (Fig. 2B). These results suggest that, within neurons, endogenous Cdh1 is indeed found in two forms, a major portion that is complexed with the APC and another portion that is not complexed with APC. In other experiments, we found that exposure of granule neurons to the Cdk inhibitor roscovitine significantly increased the proportion of Cdh1 cofractionating with Cdc27 in neurons and decreased the portion of "free" Cdh1 not in complex with the APC (Fig. $2 C, D)$. Together, these results support the conclusion that phosphorylation of Cdh1 at Cdk sites disrupts the association of Cdh1 with the APC in neurons.

We next assessed the effect of the Cdh1 phosphorylation on the subcellular localization of Cdh1 in neurons. We expressed the wild-type and phosphorylation mutant GFP-Cdh1 proteins together with $\beta$-galactosidase in granule neurons. We subjected transfected neurons to immunocytochemistry with GFP and $\beta$-galactosidase antibodies, using the latter to visualize transfected granule neurons. We also used the DNA dye bisbenzimide (Hoechst 33258) to visualize the nucleus in neurons. In agreement with our previous findings (Konishi et al., 2004; Stegmüller et al., 2006), wild-type Cdh1 was found predominantly in the nucleus (Fig. $3 A$ ). In $\sim 80 \%$ of granule neurons, Cdh1 was found exclusively in the nucleus, whereas in the remaining neurons, Cdh1 was present in both the nucleus and cytoplasm

(Fig. 3B). The 9A mutation had little effect on the subcellular localization of Cdh1 (Fig. $3 A, B$ ). In contrast, the 9D Cdh1 mutant was strikingly localized in both the cytoplasm and nucleus rather than exclusively in the nucleus (Fig. $3 A, B)$. In other experiments, we assessed the effect of expression of the neuron-specific kinase Cdk5 together with its activating partner p 25 on the subcellular localization of Cdh1 in neurons (Fig. 3C). Although wild-type Cdh1 was predominantly found exclusively in the nucleus in control neurons, Cdh1 was found in both the nucleus and cytoplasm in the majority of neurons in which p25/Cdk5 was expressed. Notably, p25/Cdk5 expression had little or no effect on the subcellular localization of the 9A and 9D Cdh1 mutant proteins in neurons. Together, these results suggest that phosphorylation of Cdh1 at Cdk sites triggers the dissociation of Cdh1 from the APC, leading to the accumulation of Cdh1 in the cytoplasm in neurons.

Having characterized the effect of Cdh1 phosphorylation at 
Cdk sites on the activity of Cdh1 and subcellular localization, we next determined the effect of Cdh1 phosphorylation on its function in the control of axon growth in primary rat cerebellar granule neurons. Although the overexpression of Cdh1 on its own does not lead to a gain of function in neurons, the expression of an RNAiresistant form of Cdh1 (Cdh1-Res) inhibits axon growth in the background of Cdh1 knockdown (Stegmüller et al., 2006). We therefore performed structure-function analyses of Cdh1-Res in this background and tested the effect of the 9A and 9D phosphorylation mutations in Cdh1 on its ability to inhibit axon growth. We found that both Cdh1-Res and Cdh1-Res 9A significantly inhibited axon growth in the background of Cdh1 RNAi (Fig. 4). Although the 9A mutant protein might have been anticipated to be more effective than wild-type Cdh1-Res at limiting axon growth because of the enhanced association of the 9A mutant with the APC, this was not observed (Fig. 4). We attribute the inability of the 9A mutant protein to further inhibit axon growth to its reduced stability, countering the positive effect of the 9A mutation on the interaction of Cdh1 with the APC, with a net result that the 9A mutant is similar or even somewhat less effective than wild-type Cdh1 in limiting axon growth. In contrast to Cdh1-Res and Cdh1-Res 9A, we found that the 9D phosphomimic Cdh1 mutant failed to inhibit axon growth (Fig. 4). Together, our results suggest that phosphorylation of Cdh1 at Cdk sites suppresses the ability of Cdh1APC to limit axon growth in primary neurons.

\section{Discussion}

In this study, we have characterized a Cdh1 phosphorylation-dependent mechanism that regulates Cdh1-APC function in the control of axon growth in mammalian neurons (see model in Fig. 4C). We found that phosphorylation of Cdh1 at Cdk sites stabilizes an inactive form of Cdh1 that fails to associate with the APC. Accordingly, phosphorylation of Cdh1 at $\mathrm{Cdk}$ sites promotes the accumulation of Cdh1 in the cytoplasm in neurons and suppresses the ability of Cdh1-APC to limit the growth of axons. These findings suggest that phosphorylation of Cdh1 may represent a critical mode of Cdh1-APC regulation in the mammalian brain.

Our findings have several important ramifications. The finding that phosphorylation of Cdh1 at Cdk sites stabilizes Cdh1 proteins suggests that phosphorylation inhibits the degradation of Cdh1 by the ubiquitin-proteasome pathway. Intriguingly, $\mathrm{Cdh} 1$ is reported to undergo autoubiquitination and consequent degradation, and remarkably the nine conserved sites of Cdkinduced phosphorylation reside within a 180 amino acid region of the N-terminal domain of Cdh1 that is required for Cdh1 autoubiquitination (Listovsky et al., 2004). Together, these ob-

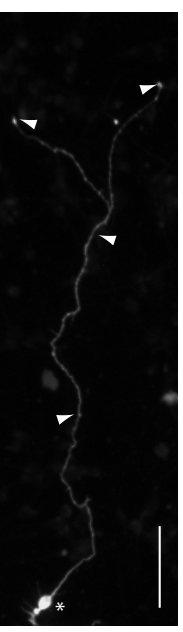

GFP

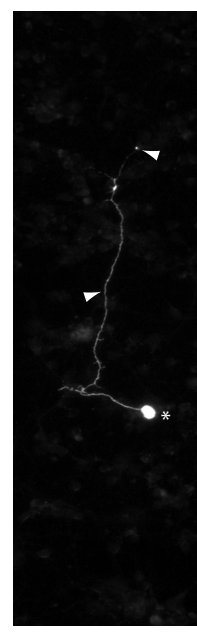

GFP-Cdh1-Res 9A

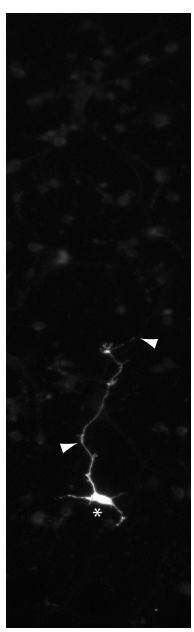

GFP-Cdh1-Res

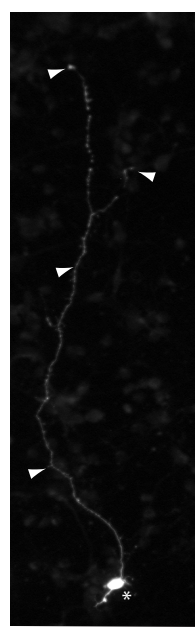

GFP-Cdh1-Res 9D
Figure 4. Phosphorylation of $C \mathrm{Ch} 1$ inhibits its ability to limit axon growth. $\boldsymbol{A}$, Granule neurons transfected with an expression plasmid and DsRed expression plasmid were subjected to immunocytochemistry using a DsRed antibody. Images of transfected background of Cdh1 RNAi. A total of 584 neurons were measured. C, A schematic of regulation of Cdh1-APC function by Cdkinduced Cdh1 phosphorylation in neurons. Active and inactive Cdh1 refer to its function in limiting axon growth.

servations support the conclusion that phosphorylation of Cdh1 at Cdk sites may inhibit the ability of Cdh1-APC to trigger Cdh1 autoubiquitination, leading to its stabilization.

The paradoxical ability of Cdk inhibition to promote Cdh1 association with the APC even as it promotes Cdh1 selfdegradation underscores the importance of gaining additional insights into the ability of Cdks to regulate Cdh1-APC activity. In future studies, it will be important to identify the Cdk that phosphorylates Cdh1 in neurons. Recent evidence suggests that Cdk5 may phosphorylate Cdh1 in cortical neurons (Maestre et al., 2008). In agreement with this finding, we also found that expression of p25/Cdk5 alters the subcellular localization in neurons. Intriguingly, Cdk5 has been implicated in neurite outgrowth (Nikolic et al., 1996; Hahn et al., 2005), making it a promising candidate for the regulation of Cdh1-APC activity in the control of axon growth. Beyond the potential role of Cdh1 phosphorylation in the developmental regulation of axon growth, the ability of 
Cdh1 phosphorylation to inhibit Cdh1-APC activity may allow harnessing of the therapeutic potential of this pathway in axonal regeneration after injury.

The abundant number of Cdk sites within Cdh1 raises the interesting possibility that processive phosphorylation of Cdh1 might regulate Cdh1 localization and stability. Although the 9D mutation altered significantly the subcellular localization of Cdh1 from exclusively nuclear to both nuclear and cytoplasmic, the 9D phosphomimic did not display an entirely cytoplasmic localization in neurons. These results suggest that phosphorylation of Cdh1 at Cdk sites may inhibit Cdh1-APC activity primarily by disrupting the association of Cdh1 with the APC. It will be interesting to determine whether a subset of Cdk phosphorylation sites specifically alters Cdh1 localization, stability, or activity or whether these phenomena are an inseparable part of the autoregulation of Cdh1-APC activity.

Our findings suggest that, just as in dividing cells, in which multiple mechanisms regulate Cdh1 activity, Cdh1 might be regulated by several mechanisms in postmitotic neurons. We have identified TGF $\beta-$ Smad 2 signaling and Cdh 1 phosphorylation as two opposing modes of regulation, the former activating and the latter inhibiting Cdh1-APC function (Stegmüller et al., 2008 and this study). It will be important to determine in future studies whether and how these mechanisms might be coordinated in neurons. As parallels are established for the regulation of Cdh1 in dividing cells and postmitotic neurons, the intricate regulation of Cdh1-APC activity in proliferating cells may prove equally exquisite in the mammalian nervous system.

\section{References}

Almeida A, Bolaños JP, Moreno S (2005) Cdh1/Hct1-APC is essential for the survival of postmitotic neurons. J Neurosci 25:8115-8121.

Gaudillière B, Konishi Y, de la Iglesia N, Yao G, Bonni A (2004) A CaMKIINeuroD signaling pathway specifies dendritic morphogenesis. Neuron 41:229-241.

Hahn CM, Kleinholz H, Koester MP, Grieser S, Thelen K, Pollerberg GE (2005) Role of cyclin-dependent kinase 5 and its activator P35 in local axon and growth cone stabilization. Neuroscience 134:449-465.

Ikeuchi Y, Stegmüller J, Netherton S, Huynh MA, Masu M, Frank D, Bonni S, Bonni A (2009) A SnoN-Ccdl pathway promotes axonal morphogenesis in the mammalian brain. J Neurosci 29:4312-4321.

Jaspersen SL, Charles JF, Morgan DO (1999) Inhibitory phosphorylation of the APC regulator Hctl is controlled by the kinase Cdc28 and the phosphatase Cdc14. Curr Biol 9:227-236.

Juo P, Kaplan JM (2004) The anaphase-promoting complex regulates the abundance of GLR-1 glutamate receptors in the ventral nerve cord of $C$. elegans. Curr Biol 14:2057-2062.

Kim AH, Bonni A (2007) Thinking within the D box: initial identification of
Cdh1-APC substrates in the nervous system. Mol Cell Neurosci 34:281-287.

Kim AH, Puram SV, Bilimoria PM, Ikeuchi Y, Keough S, Wong M, Rowitch D, Bonni A (2009) A centrosomal Cdc20-APC pathway controls dendrite morphogenesis in postmitotic neurons. Cell 136:322-336.

Konishi Y, Stegmüller J, Matsuda T, Bonni S, Bonni A (2004) Cdh1-APC controls axonal growth and patterning in the mammalian brain. Science 303:1026-1030.

Kramer ER, Scheuringer N, Podtelejnikov AV, Mann M, Peters JM (2000) Mitotic regulation of the APC activator proteins Cdc20 and Cdh1. Mol Biol Cell 11:1555-1569.

Lasorella A, Stegmüller J, Guardavaccaro D, Liu G, Carro MS, Rothschild G, de la Torre-Ubieta L, Pagano M, Bonni A, Iavarone A (2006) Degradation of Id 2 by the anaphase-promoting complex couples cell cycle exit and axonal growth. Nature 442:471-474.

Li M, Shin YH, Hou L, Huang X, Wei Z, Klann E, Zhang P (2008) The adaptor protein of the anaphase promoting complex Cdh1 is essential in maintaining replicative lifespan and in learning and memory. Nat Cell Biol 10:1083-1089.

Listovsky T, Oren YS, Yudkovsky Y, Mahbubani HM, Weiss AM, Lebendiker M, Brandeis M (2004) Mammalian Cdh1/Fzr mediates its own degradation. EMBO J 23:1619-1626.

Maestre C, Delgado-Esteban M, Gomez-Sanchez JC, Bolaños JP, Almeida A (2008) Cdk5 phosphorylates Cdh1 and modulates cyclin B1 stability in excitotoxicity. EMBO J 27:2736-2745.

Nikolic M, Dudek H, Kwon YT, Ramos YF, Tsai LH (1996) The cdk5/p35 kinase is essential for neurite outgrowth during neuronal differentiation. Genes Dev 10:816-825.

Peters JM (2006) The anaphase promoting complex: a machine designed to destroy. Nat Rev Mol Cell Biol 7:644-656.

Pot I, Bonni S (2008) SnoN in TGF-beta signaling and cancer biology. Curr Mol Med 8:319-328.

Stegmüller J, Bonni A (2005) Moving past proliferation: new roles for Cdh1-APC in postmitotic neurons. Trends Neurosci 28:596-601.

Stegmüller J, Konishi Y, Huynh MA, Yuan Z, Dibacco S, Bonni A (2006) Cell-intrinsic regulation of axonal morphogenesis by the Cdh1-APC target SnoN. Neuron 50:389-400.

Stegmüller J, Huynh MA, Yuan Z, Konishi Y, Bonni A (2008) TGFbetaSmad2 signaling regulates the Cdh1-APC/SnoN pathway of axonal morphogenesis. J Neurosci 28:1961-1969.

Sullivan M, Morgan DO (2007) Finishing mitosis, one step at a time. Nat Rev Mol Cell Biol 8:894-903.

van Roessel P, Elliott DA, Robinson IM, Prokop A, Brand AH (2004) Independent regulation of synaptic size and activity by the anaphasepromoting complex. Cell 119:707-718.

Zachariae W, Shin TH, Galova M, Obermaier B, Nasmyth K (1996) Identification of subunits of the anaphase-promoting complex of Saccharomyces cerevisiae. Science 274:1201-1204.

Zachariae W, Schwab M, Nasmyth K, Seufert W (1998) Control of cyclin ubiquitination by Cdk-regulated binding of Hctl to the anaphase promoting complex. Science 282:1721-1724.

Zhou Y, Ching YP, Chun AC, Jin DY (2003) Nuclear localization of the cell cycle regulator Cdh1 and its regulation by phosphorylation. J Biol Chem 278:12530-12536. 